Proceedings

\title{
The Effect of Different Titers of Blood-Grouping Reagents on Proliferation and Apoptosis of Bone Marrow Derived Mesenchymal Stem Cells ${ }^{+}$
}

\author{
Habibe Meltem Özgüner 1,2,*, Yasin Köksal 2, Mücahit Aydoğdu ${ }^{1}$, Merve Ünver ${ }^{1}$, Tuğçe Uykun ${ }^{1}$, \\ GökçeAkay ${ }^{1}$ and Tuba Özdemir Sancı ${ }^{1}$ \\ 1 Department of Histology and Embryology, Faculty of Medicine, Ankara Yıldırım Beyazıt University, \\ 06800 Ankara, Turkey; m.aydogdugs@hotmail.com (M.A.); merveunver35@gmail.com (M.Ü.); \\ tugceuykunn@gmail.com (T.U.); gokcebiyo@gmail.com (G.A.); tubaaozdemirr@gmail.com (T.Ö.S.) \\ 2 Ankara Child Health and Disease Hematology Oncology Training and Research Hospital, 06080 Ankara, \\ Turkey; yakkoksal@mynet.com \\ * Correspondence: mozguner@hotmail.com \\ † Presented at the 2nd International Cell Death Research Congress, Izmir, Turkey, 1-4 November 2018. \\ Published: 5 December 2018
}

\begin{abstract}
Hematopoetic Stem Cell Transplantation (HSCT) has benefical effects for the treatment of leukemia but one of the adverse effect is Graft versus Host Disease (GVHD) that causes high mortality and morbidity. Mesenchymal Stem Cells (MSCs) are one of the promising tools which are used for the treatment of steroid refractory GVHD. The success of cellular therapies depends on so many factors associated with the donor and host characteristics. We designed a study concerning that blood group reagents at different titres can affect the survival of MSCS in blood as they were injected intravenously when used for the treatment of GVHD. We concluded that proliferation rates of MSCs are decreasing in relation with the high reagent titres. Flow-cytometric analysis also revealed that high titers of reagent in culture medium could lead to the induction of apoptotic pathways in MSCs. For individualized treatment strategies, donor and host characteristics must be taken in to consideration in details and experimental studies in cell culture laboratories could be essential to gain insight into clinical trials.
\end{abstract}

Keywords: Hematopoetic Stem Cell Transplantation (HSCT); Graft Versus Host Disease (GVHD); Mesenchymal Stem Cells (MSCs); apoptosis

\section{Introduction}

Hematopoetic Stem Cell Transplantation (HSCT) is a lifesaving therapeutic option for hematopoetic malignancies especially in children. Therefore, donor-versus-host alloreactivity is a serious and potentially life-threatening complication as donor lymphocytes could destroy host organs [1]. High dose steroids are the first choice of treatment in Graft versus Host Disease (GVHD), however different therapeutic options targeting immun cells have also been demonstrated in steroid refractory GVHD [1]. MSCs are the main cells used in regenerative medicine, in addition to their regenerative properties, they have also immunosuppressive properties in vitro and in vivo [2]. In recent years, phase I-II studies have suggested that MSCs can be used as a therapeutics agent for the prevention and/or treatment for severe GVHD [3]. In our HSCT Unit, even though we use the standard protocol for the infusion of MSCs, steroid refractory GVHD patients had different remission (complete -partial or no remission) profiles. In this study, we aimed to investigate whether the different remission profiles have a relation with the recipients' blood group antigens and 
whether different blood group antigens (the difference between the blood groups of the donor and the patient) can induce the apoptosis of MSCs and change directly the survival, and indirectly the effectiveness of MSCs in the blood stream.

\section{Materials and Methods}

Bone Marrow samples of three healthy donors were included in the study and informed consent was obtained from all donors, and their legal guardians for HSCT. This study was approved by the local ethical committee.

Isolation and Culture of Human BM- Derived MSCs; BM samples were diluted with Dulbecco's PBS (Biochrom, Berlin, Germany) after the plasma collection and layered on Biocoll $(1.077 \mathrm{~g} / \mathrm{mL})$ separating solution (Biochrom, Berlin, Germany) (1:1) and centrifuged at $2200 \mathrm{rpm}$ for $20 \mathrm{~min}$. The supernatant was discarded, and the pellet was resuspended in $10 \mathrm{~mL}$ DMEM-LG, 10\% FBS, and 1\% penicillin-streptomycin (Biochrom, Berlin, Germany) and at least $20 \times 10^{6}$ cell were seeded in 75 $\mathrm{cm}^{2}$ culture flasks. Flasks were kept at $37^{\circ} \mathrm{C}$ in a humidified atmosphere containing $5 \% \mathrm{CO}_{2}$ (Galaxy 170R incubator, Eppendorf Company, Hamburg, Germany). After $72 \mathrm{~h}$ of incubation, non-adherent cells were removed. The cells were trypsinized when adherent cells were reached $70 \%$ to $80 \%$ confluency. Then the cells were seeded at a concentration of $25 \times 10^{4} \mathrm{cells} /$ well in 6 well plates and the culture medium was supplemented with blood group reagents at that time point of the experiment. After $24 \mathrm{~h}$ of incubation, the proliferation percentages of cells in each well were evaluated. Experiment groups including blood groups of healthy donors and selected blood grouping reagent (Anti-A) titers are presented in Table 1.

Table 1. Proliferation rates of MSCs after $24 \mathrm{~h}$ of culture with different titers of blood grouping reagent.

\begin{tabular}{lccccc}
\hline & \multirow{2}{*}{ Blood Group of Donor } & Control & Anti-A & Anti-A & Anti-A \\
& & (No Antibody) & $\mathbf{6} \boldsymbol{\mu L}$ & $\mathbf{1 2} \boldsymbol{\mu L}$ & $\mathbf{2 4} \boldsymbol{\mu L}$ \\
\hline Donor \#1 & AB Rhpositive & $95 \%$ & $90 \%$ & $85 \%$ & $85 \%$ \\
Donor \#2 & A Rhpositive & $95 \%$ & $60 \%$ & $50 \%$ & $40 \%$ \\
Donor \#3 & 0 Rhpositive & $95 \%$ & $75 \%$ & $60 \%$ & $30 \%$ \\
\hline
\end{tabular}

Apoptosis determination by Annexin V/PI in flow-cytometry; Flow cytometric analysis were performed by using FITC Annexin V apoptosis detection kit with Propidium Iodide (PI). Cells were acquired on a Beckmann Coulter Navios Flow-Cytometer (Beckmann Coulter, Brea, CA, USA) using Kaluza version 1.2 software. Analysis were documented according to the literature.

\section{Results}

Proliferation rates of MSCs after $24 \mathrm{~h}$ of culture with different titers of blood grouping reagent were represented in Table 1. Microscopic images from representative samples of MSCs derived from 3 donors after $24 \mathrm{~h}$ of incubation with different titers of Anti-A reagent are shown in Figure 1.

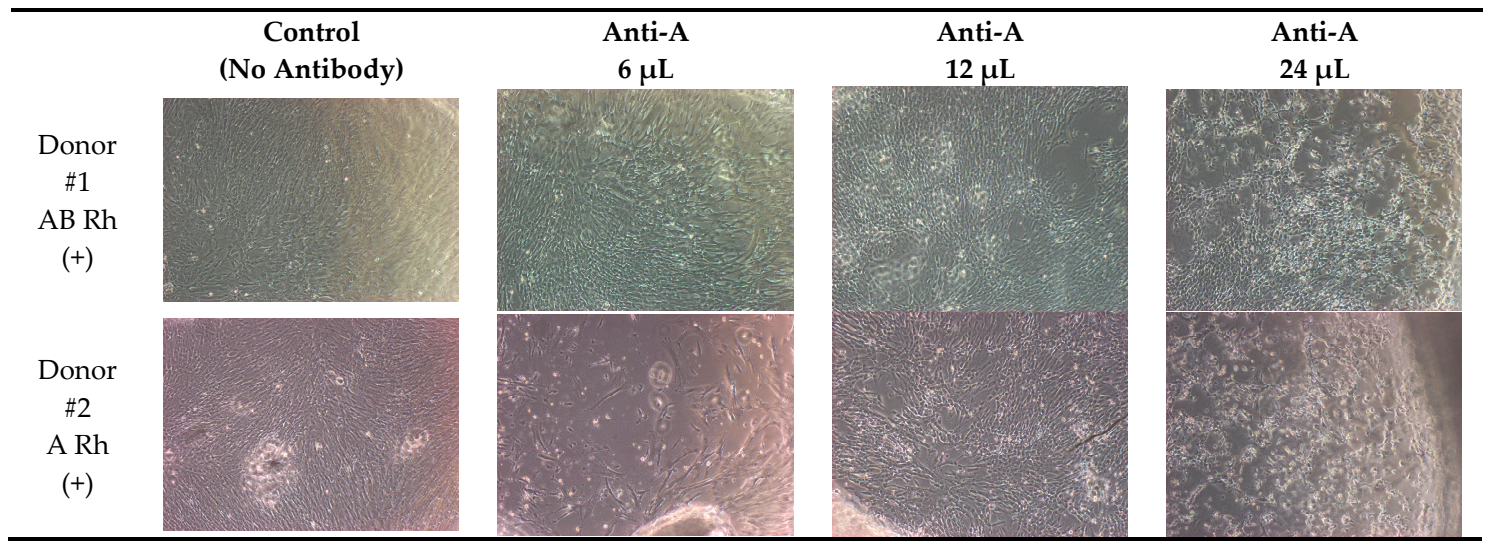




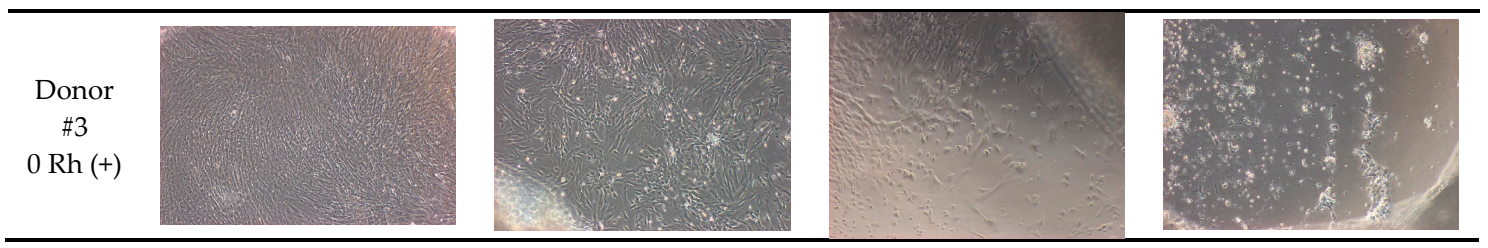

Figure1. Proliferation assay of MSCs under light microscope ( $\times 10$ magnification).

Flow-cytometric data are illustrated in Figure 2. The percentage of late apoptotic cells was $62 \%$ in control well and the percentage of late apoptotic cells were $72 \%, 78 \%$ and $89 \%$ for the culture of MSCs with $6 \mu \mathrm{L}, 12 \mu \mathrm{L}$ and $24 \mu \mathrm{L}$ of Anti-A reagent respectively for $24 \mathrm{~h}$.

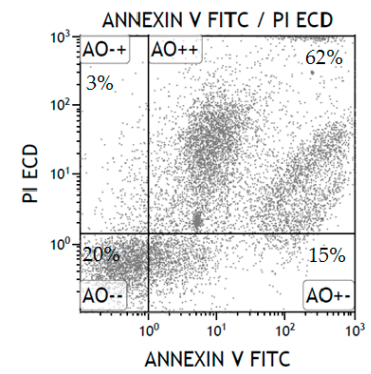

(a)

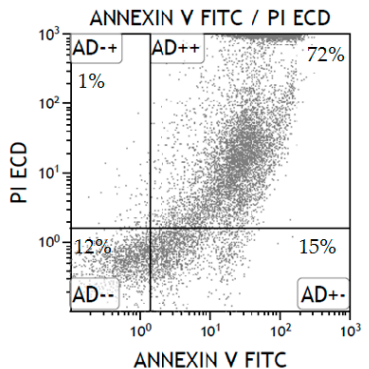

(b)

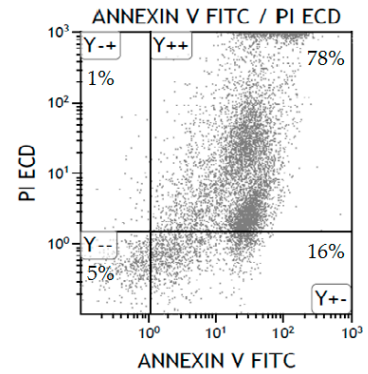

(c)

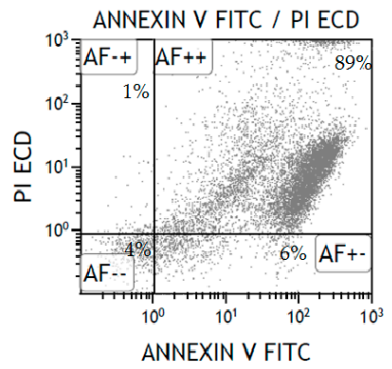

(d)

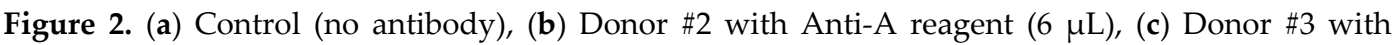
Anti-A reagent $(12 \mu \mathrm{L}),(\mathrm{d})$ Donor \#1 Anti-A $(24 \mu \mathrm{L})$.

\section{Discussion}

MSCs are the representative of adult type of multipotent cells and appear to be a promising therapy for GVHD, so most of the laboratory studies are focused on the ex-vivo expansion of these cells and culture conditions, besides, most of the clinical trials are focused on the cell dose, time intervals of the cell infusion or duration of the treatment [3]. Individualized treatment strategies are important concerning the GVHD subtypes, pathological mechanisms and stages, so we also experienced different remission profiles during the GVHD treatment. For this reason, we designed a study with BM derived MSCs to investigate whether in-vivo conditions including blood grouping reagents can have an effect on MSCs. The results of the proliferation assays revealed that the most effected MSCs were donor \#3 (0 blood group) with the highest Anti-A reagent $(24 \mu \mathrm{L})$ and the proliferation rate was the lowest $30 \%$. To define whether the low proliferation rate has any relation with the induction of apoptotic pathways in MSCs, we performed flow-cytometric analysis.

Apoptosis is accompanied by numerous morphological characteristics and also nuclear condensation, DNA fragmentation, externalization of phosphatidylserine (PS) and activation of caspases [4]. In our study, we demonstrated increased percentage of late apoptotic cells related with the increased titers of blood grouping reagent in the culture. Therefore, the percentage of apoptotic cells were quite high even in control group (62\%). We predicted that could be the result of contact inhibition in culture flask as control group had a high proliferation rate than the others.

This study was the first to investigate whether different titres of blood group reagents had an effect on MSCs biology and in-vivo survival of MSCs after given intravenously. These results predicted that blood group antibodies in-vivo could effect the survival of MSCs and induce apoptotic pathways in these cells which could also decrease their efficacy in cellular therapies. As a conclusion, understanding ex-vivo and in-vivo behaviours of MSCs under different biological conditions can help to overcome so many problems before their wide clinical application.

Author Contributions: H.M.Ö. and Y.K., designed the study; H.M.Ö. wrote the manuscript; M.A., M.Ü., T.U., G.A., and Y.K. performed the experiments. All authors read approved the final manuscript. 


\section{References}

1. Sullivan, K.M. Graft-vs.-host disease. In Thomas' Hematopoietic Cell Transplantation; Blume, K.G., Forman, S.J., Appelbaum, F.R., Eds.; Blackwell Publishing Ltd.: Oxford, UK, 2004; pp. 635-664.

2. De Miguel, M.P.; Fuentes-Julián, S.; Blázquez-Martínez, A.; Pascual, C.Y.; Aller, M.A.; Arias, J.; Arnalich-Montiel, F. Immunosuppressive properties of mesenchymal stem cells: Advances and applications. Curr. Mol. Med. 2012, 12, 574-591.

3. Le Blanc, K.; Frassoni, F.; Ball, L.; Locatelli, F.; Roelofs, H.; Lewis, I.; Lanino, E.; Sundberg, B.; Bernardo, M.E.; Remberger, M.; et al. Mesenchymal stem cells for treatment of steroid-resistant, severe, acute graft-versushostdisease: A phase II study. Lancet 2008, 371, 1579-1586.

4. Conor, M.H.; Hollville, E.; Seamus, J.M. Measuring apoptosis by microscopy and flow cytometry. Methods 2013, 61, 90-97.

(C) 2018 by the authors. Licensee MDPI, Basel, Switzerland. This article is an open access article distributed under the terms and conditions of the Creative Commons Attribution (CC BY) license (http://creativecommons.org/licenses/by/4.0/). 